References:

[1] Martyna TS,Hanna SS, Marek F. Clinical and immunological characteristics of Polish patients with systemic lupus erythematosus. Adv Clin Exp Med. 2018;27(1):57-61

[2] Maloney K C, Ferguson T S, Stewart H D, et al. Clinical and immunological characteristics of 150 systemic lupus erythematosus patients in Jamaica: A comparative analysis[J]. Lupus, 2017, 26(13):961203317707828.

[3] Ching K H, Burbelo P D, Christopher T, et al. Two Major Autoantibody Clusters in Systemic Lupus Erythematosus[J]. PLoS ONE, 2012, 7(2):e32001.

Disclosure of Interests: None declared

DOI: 10.1136/annrheumdis-2020-eular.4871

\section{AB0447 IRREVERSIBLE ORGAN DAMAGE IN PERI- AND POSTMENOPAUSAL WOMEN WITH SYSTEMIC LUPUS ERYTHEMATOSUS IN REMISSION}

S. Shkireeva ${ }^{1}$, E. Zotkin ${ }^{1}$, O. Lesnyak ${ }^{2} .{ }^{1}$ Science and Research Institution of Rheumatology named after V.A. Nasonova, Moscow, Russian Federation

${ }^{2}$ North-Western State Medical University named after I.I. Mechnikov, SaintPetersburg, Russian Federation

Background: Although the survival of patients with systemic lupus erythematosus (SLE) has improved, irreversible organ damage remains a critical concern. Long-standing inflammation, drug-related side effects and comorbidities may eventually cause permanent organ damage even in remission [1].

Objectives: To describe irreversible organ damage in peri- and postmenopausal women with SLE in remission and low disease activity, to find predictors of damage progression.

Methods: 234 peri- and postmenopausal women with SLE were included (mean age $49,94 \pm 9,1$ years) in our study. All women were under outpatient observation in St.Petersburg State Clinical Rheumatology Hospital \#25 (Russia). Mean disease duration was $8,9 \pm 7,5$ years. We analyzed treatment regimens and doses of glucocorticoids (GC) based on source medical documents. To assess disease activity, we used SLEDAI-2K and LLDAS. To assess organ damage, we used SLICC damage index (SDI).

Results: $94,3 \%$ of women have been taking GC during our study. Median of maintenance dose was $12,5 \mathrm{mg}$ per day. Almost a half of all women $(44,8 \%$, $\mathrm{n}=105$ ) in our study were postmenopausal (mean duration of menopause was $11,1 \pm 7,1$ years). A half of all patients had low disease activity $(44,4 \%, n=111)$ or were in remission $(18,8 \%, n=44)$ according to SLEDAI-2K. $26,5 \%(n=65)$ of patients had all 5 criteria and $45,1 \%(n=115)$ of patients had 4 of 5 criteria according to LLDAS. Critical organ damage (SDI>4) was observed in $68,8 \%$ $(n=161)$ of women with SLE. Moderate $(1 \leq S D I \leq 4)$ and low $(S D I=1)$ damage had $25,6 \%(n=60)$ and $6,4 \%(n=15)$ of patients respectively. Musculoskeletal damage was on the first place among others: $37,6 \%(n=88)$ of patients had osteoporosis with fractures and $34,2 \%(n=80)$ had muscle weakness. In 75 women with SLE of osteoporotic fractures occurred in remission or low disease activity (LLDAS). Progression of irreversible organ damage in remission or low disease activity (LLDAS) had $62 \%(n=145)$ of women with SLE. Multifactorial logistic regression analysis of factors associated with organ damage in SLE showed that only patients age $(p=0.013215)$, cumulative dose of $G C$ $(p=0.000047)$ and therapy with cyclophosphamide $(p=0.041505)$ were statistically significant.

Conclusion: Progression of irreversible organ damage in peri- and postmenopausal women with SLE may occur despite remission or low disease activity. There are no doubts that organ damage accrual is associated with CG therapy. Correction of GC dose or discontinuation of GC treatment in remission can predict organ damage accrual in SLE including osteoporosis and osteoporotic fractures.

References:

[1] Frodlund M, Reid S, Wetterö J, Dahlström Ö, Sjöwall C, Leonard D. The majority of Swedish systemic lupus erythematosus patients are still affected by irreversible organ impairment: factors related to damage accrual in two regional cohorts. Lupus. 2019;28(10):1261-1272. doi:10.1177/0961203319860198

Disclosure of Interests: None declared

DOI: 10.1136/annrheumdis-2020-eular.2420

\section{$\mathrm{AB0448}$ \\ IMPACT OF REMISSION ON DAMAGE ACCRUAL IN SYSTEMIC LUPUS ERYTHEMATOSUS (SLE) PATIENTS: A SYSTEMATIC LITERATURE REVIEW (SLR)}

M. F. Ugarte-Gil ${ }^{1,2}$, C. Mendoza Pinto ${ }^{3}$, G. Pons-Estel ${ }^{4}$, C. Reategui Sokolova ${ }^{1,5}$, R. Van Vollenhoven ${ }^{6}$, G. Bertsias ${ }^{7}$, G. S. Alarcon ${ }^{8,9}$, B. Pons-Estel ${ }^{4}{ }^{1}$ Hospital $^{2}$ Guillermo Almenara Irigoyen. EsSalud, Lima, Peru; ${ }^{2}$ Universidad Científica del Sur, Lima, Peru; ${ }^{3}$ Benemérita Universidad Autónoma de Puebla, Puebla, Mexico; ${ }^{4}$ Centro Regional de Enfermedades Autoinmunes y Reumáticas

\begin{tabular}{|c|c|c|c|c|c|c|}
\hline Authors & $\begin{array}{l}\text { Country/ } \\
\text { Region }\end{array}$ & Patients & Remission & $\begin{array}{c}\text { Achieving } \\
\text { remission } \\
(\%)\end{array}$ & $\begin{array}{l}\text { Damage: } \\
\text { remission vs not } \\
\text { remission }\end{array}$ & NOS \\
\hline Zen et al & Italy & 224 & $\begin{array}{c}\text { SLEDAI }=0 \\
\text { Serologic }=\text { allowed } \\
\text { PGA }=\text { NR } \\
\text { PDN } \leq 5 \\
\text { IS }=\text { Yes } \\
\text { AM }=\text { Yes } \\
\text { Duration }=5 \text { years }\end{array}$ & $37.5 \%$ & $\begin{array}{c}\text { Unremitted } \\
\text { disease } \\
\text { OR=2.53; } \\
p=0.008\end{array}$ & 7 \\
\hline Tani et al & Italy & 115 & $\begin{array}{c}\text { SLEDAI }=0 \\
\text { Serologic } \\
\text { PGA }=\text { NR } \\
\text { PDN } \leq 5 \\
\text { IS }=\text { Yes } \\
\text { AM }=\text { Yes } \\
\text { Duration=NR }\end{array}$ & $49.6 \%$ & $\begin{array}{r}\Delta \text { SLICC } 0.12 \text { vs } \\
0.48, p=0.018\end{array}$ & 8 \\
\hline $\begin{array}{l}\text { Ugarte- } \\
\quad \text { Gil } \\
\text { et al }\end{array}$ & $\begin{array}{l}\text { Latin } \\
\text { America }\end{array}$ & 1,350 & $\begin{array}{c}\text { SLEDAI }=0 \\
\text { Serologic }=\text { not allowed } \\
\text { PGA }=\text { NR } \\
\text { PDN } \leq 5 \\
\text { IS }=\text { Yes } \\
\text { AM }=\text { Yes } \\
\text { Duration=NR }\end{array}$ & $20.2 \%$ & $\begin{array}{c}\text { New damage } \\
\text { HR } 0.60 \\
p=0.0042 \\
\text { Severe new } \\
\text { damage } \\
\text { HR } 0.32 \\
p=0.0033\end{array}$ & 8 \\
\hline $\begin{array}{l}\text { Tsang-A- } \\
\text { Sjoe } \\
\text { et al }\end{array}$ & Netherlands & 224 & $\begin{array}{c}\text { SLEDAI }=0 \\
\text { Serologic }=\text { allowed } \\
P G A=N R \\
P D N \leq 5 \\
I S=Y e s \\
A M=Y e s \\
\text { Duration }>5 \text { years }\end{array}$ & $37.6 \%$ & $\begin{array}{r}\mathrm{OR}=0.20 \\
p=0.001\end{array}$ & 8 \\
\hline Mok et al & China & 769 & $\begin{array}{c}\text { SLEDAl }=0 \\
\text { Serologic }=\text { Allowed } \\
P G A<0.5 \\
P D N \leq 5 \\
I S=Y e s \\
A M=\text { Yes } \\
\text { Duration } \geq 5 \text { years }\end{array}$ & $25.1 \%$ & $\begin{array}{c}\text { Not being on } \\
\text { remission } \\
\text { for at least } 5 \\
\text { years } \\
\text { OR: } 2.42 ; \\
\text { p }<0.001\end{array}$ & 7 \\
\hline $\begin{array}{r}\text { Tselios } \\
\text { et al }\end{array}$ & Canada & 267 & $\begin{array}{c}\text { SLEDAI }=0 \\
\text { Serologic }=\text { Allowed } \\
\text { PGA }=\text { NR } \\
\text { PDN no restriction } \\
\text { IS no restriction } \\
\text { AM }=\text { Yes } \\
\text { Duration } \geq 5 \text { years }\end{array}$ & $10.1 \%$ & $\begin{array}{l}\text { SDI after } 10 \\
\text { years: } 0.96 \\
\text { vs } 1.53 \text { (not } \\
\text { on remission, } \\
\text { not on LDAS), } \\
\text { p for trend } \\
=0.029\end{array}$ & 7 \\
\hline Petri et al & US & 1,356 & $\begin{array}{c}\text { SLEDAI }=0 \\
\text { Serologic }=\text { NR } \\
\text { PGA }<0.5 \\
\mathrm{PDN} \leq 5 \\
\text { IS }=\text { Yes } \\
\mathrm{AM}=\text { Yes } \\
\text { Duration }=\mathrm{NR}\end{array}$ & NR & $\begin{array}{c}\text { Rate ratio per } \\
\text { percentage of } \\
\text { follow-up } \\
R R(<25 \%)=0.54 ; \\
p<0.0001 \\
R R(25- \\
49 \%)=0.47 ; \\
p<0.0001 \\
R R(50- \\
74 \%)=0.43 ; \\
p<0.0001 \\
R R(>75 \%)=0.45 ; \\
p=0.0019\end{array}$ & 7 \\
\hline $\begin{array}{r}\text { Golder } \\
\text { et al }\end{array}$ & Asia Pacific & 1,707 & $\begin{array}{c}\text { SLEDAI }=0 \\
\text { Serologic=Yes } \\
P G A<0.5 \\
P D N \leq 5 \\
\text { IS }=\text { Yes } \\
\text { AM=Yes } \\
\text { Duration= NR }\end{array}$ & $35.8 \%$ & $\begin{aligned} H R & =0.55 \\
p & <0.0001\end{aligned}$ & 6 \\
\hline
\end{tabular}

NR: Not recorded. OR: Odds Ratio. HR: Hazard ratio. RR: Rate ratio

(GO-CREAR), Rosario, Argentina; ${ }^{5}$ Universidad San Ignacio de Loyola, Unidad de Investigación para la Generación y Síntesis de Evidencias en Salud, Lima, Peru; ${ }^{6}$ Amsterdam UMC, Amsterdam, Netherlands; ${ }^{7}$ University of Crete, Heraklion, Greece; ${ }^{8}$ University of Alabama at Birmingham, Alabama, United States of America; ${ }^{9}$ Universidad Peruana Cayetano Heredia, Lima, Peru

Background: Treat-to-target strategy with remission as the target has been proposed for the management of SLE. However, there is not a uniform definition of remission.

Objectives: To determine the protective value of remission state on organ damage accrual in SLE patients through a SLR

Methods: Two independent reviewers identified studies in Medline and Cochrane Library. Data on remission definitions and rates as well as damage accrual (assessed by the SLICC/ACR damage index [SDI]) were extracted. Definitions of 
remission included disease activity indices (SLEDAI and its variants and PGA), serological activity, prednisone (PDN) daily dose (mg/day), immunosuppressive (IS) drugs, antimalarial (AM) use and duration of remission. The quality of the studies was evaluated with the Newcastle-Otawa Scale (NOS).

Results: Eight manuscripts were included comprising more than 6,000 patients from America, Europe and Asia Pacific. All the studies were longitudinal. The majority of the studies reached more than seven out nine points in the NOS. Remission rates ranged between 10 and almost $50 \%$; they tend to be lower in America as compared to Asia Pacific and Europe. All definitions required a clinical SLEDAI=0, and allowed antimalarial use. However, there were differences regarding the inclusion of serological activity, PGA, prednisone or immunosuppressive drug use as well as minimum remission duration required. Even less stringent definition of remission prevented damage accrual. The risk of damage accrual was two to five-fold lower in those patients on remission.

Conclusion: In SLE patients, achieving remission, even with less stringent definitions, prevented damage accrual.

Disclosure of Interests: : Manuel F. Ugarte-Gil Grant/research support from: Jannsen, Pfizer, Claudia Mendoza Pinto: None declared, Guillermo Pons-Estel Grant/research support from: JANSSEN and GSK, Consultant of: JANNSEN, GSK and SANOFI, Speakers bureau: PFIZER, JANNSEN and GSK, Cristina Reategui Sokolova: None declared, Ronald van Vollenhoven Grant/research support from: AbbVie, Arthrogen, Bristol-Myers Squibb, GlaxoSmithKline, Lilly, Pfizer, and UCB, Consultant of: AbbVie, AstraZeneca, Biotest, Bristol-Myers Squibb, Celgene, GSK, Janssen, Lilly, Medac, Merck, Novartis, Pfizer, Roche, and UCB, George Bertsias Grant/research support from: GSK, Consultant of: Novartis, Graciela S Alarcon: None declared, Bernardo Pons-Estel Grant/ research support from: GSK, Janssen, Consultant of: GSK, Janssen, Speakers bureau: GSK, Janssen

DOI: 10.1136/annrheumdis-2020-eular.2037

\section{AB0449 IMPACT OF REMISSION AND LOW DISEASE ACTIVITY (LDA) ON HEALTH-RELATED QUALITY OF LIFE (HRQOL) IN SYSTEMIC LUPUS ERYTHEMATOSUS (SLE) PATIENTS: A SYSTEMATIC LITERATURE REVIEW (SLR)}

M. F. Ugarte-Gil ${ }^{1,2}$, C. Mendoza Pinto ${ }^{3,4}$, C. Reategui Sokolova ${ }^{1,5}$, G. PonsEstel $^{6}$, R. Van Vollenhoven ${ }^{7}$, G. Bertsias ${ }^{8}$, G. S. Alarcon ${ }^{9}$, B. Pons-Estel ${ }^{6}$. ${ }^{1}$ Hospital Guillermo Almenara Irigoyen, Essalud, Lima, Peru; ${ }^{2}$ Universidad Científica del Sur, Lima, Peru; ${ }^{3}$ Benemérita Universidad Autónoma de Puebla, Puebla, Mexico; ${ }^{4}$ Instituto Mexicano de Seguridad Social, Puebla, Mexico; ${ }^{5}$ Universidad San Ignacio de Loyola, Lima, Peru; ${ }^{6}$ Centro Regional de Enfermedades Autoinmunes y Reumáticas (GO-CREAR), Rosario, Argentina; ${ }^{7}$ Amsterdam UMC, Amsterdam, Netherlands; ${ }^{8}$ University of Crete, Heraklion, Greece; ${ }^{9}$ University of Alabama at Birmingham, Alabama, United States of America

Background: Treat-to-target strategy has been proposed in SLE. Achieving remission/LDAS should prevent damage, reduce mortality and improve HRQoL. Objectives: To determine the protective value of remission or LDA states on HRQoL in SLE using a SLR.

Methods: Two independent reviewers identified studies in Medline and Cochrane library and extracted data on remission, LDA and HRQoL. Remission and LDA definitions included disease activity (SLEDAI and its variants, SLAM and PGA), serological activity, new organ/system, prednisone (PDN) dose (mg/day), immunosuppressives (IS) drugs, antimalarial (AM) use and remission duration. The quality of the studies was assessed with the Newcastle-Otawa Scale (NOS).

Results: Three manuscripts (1059 patients) for remission and 4 (2385 patients) for LDA were included (America, Europe \& Asia Pacific). All the studies reached seven out of nine NOS points. Remission rates ranged $25-39 \%$; and LDA: $42-62 \%$. Even less stringent remission or LDA definitions predicted/were associated with a better HRQoL. Physical rather than mental domains were more associated with remission or LDA.

Conclusion: In SLE patients, achieving remission or LDA, is associated with a better HRQoL.

Disclosure of Interests: Manuel F. Ugarte-Gil Grant/research support from: Jannsen, Pfizer, Claudia Mendoza Pinto: None declared, Cristina Reategui Sokolova: None declared, Guillermo Pons-Estel Grant/research support from: JANSSEN and GSK, Consultant of: JANNSEN, GSK and SANOFI, Speakers bureau: PFIZER, JANNSEN and GSK, Ronald van Vollenhoven Grant/research support from: AbbVie, Arthrogen, Bristol-Myers Squibb, GlaxoSmithKline, Lilly, Pfizer, and UCB, Consultant of: AbbVie, AstraZeneca, Biotest, Bristol-Myers Squibb, Celgene, GSK, Janssen, Lilly, Medac, Merck, Novartis, Pfizer, Roche, and UCB, George Bertsias Grant/research support from: GSK, Consultant of: Novartis, Graciela S
Table 2. Association between LDA and HRQoL

\begin{tabular}{|c|c|c|c|c|c|}
\hline Authors & $\begin{array}{l}\text { Country/ } \\
\text { Region }\end{array}$ & Patients & LDA & LDA (\%) & $\begin{array}{l}\text { Domains positively associ- } \\
\text { ated or predicted by LDAS }\end{array}$ \\
\hline Golder et $\mathrm{al}^{\star}$ & Asia Pacific & 1422 & $\begin{array}{c}\text { SLEDAI } \leq 4 \\
\text { PGA } \leq 1 \\
\text { PDN } \leq 7.5 \\
\text { IS }=\text { Yes } \\
\text { AM=Yes } \\
\text { New manifesta- } \\
\text { tions: No } \\
\text { Duration=NR }\end{array}$ & 42.0 & $\begin{array}{l}\text { SF-36: } \\
\text { Role physical, bodily pain, } \\
\text { general health, vitality, } \\
\text { social functioning, role } \\
\text { emotional, mental health, } \\
\text { PCS and MCS }\end{array}$ \\
\hline $\begin{array}{l}\text { Ugarte-Gil et } \\
\text { al\# }\end{array}$ & USA & 483 & $\begin{array}{c}\mathrm{SLAM} \leq 3 \\
\mathrm{PGA}=\mathrm{NR} \\
\mathrm{PDN} \leq 7.5 \\
\text { IS=No } \\
\text { AM=Yes } \\
\text { New manifesta- } \\
\text { tions: NR } \\
\text { Duration=NR }\end{array}$ & NR & $\begin{array}{l}\text { SF-36: } \\
\text { Physical function, role } \\
\text { physical, bodily pain, } \\
\text { general health, vitality, } \\
\text { social functioning, role } \\
\text { emotional, mental health, } \\
\text { PCS and MCS }\end{array}$ \\
\hline $\begin{array}{l}\text { Ugarte-Gil et } \\
\text { al\# }\end{array}$ & Peru & 243 & $\begin{array}{c}\text { SLEDAI } \leq 4 \\
\text { PGA=NR } \\
\text { PDN } \leq 7.5 \\
\text { IS }=\text { Yes } \\
\text { AM=Yes } \\
\text { New manifesta- } \\
\text { tions: NR } \\
\text { Duration=NR }\end{array}$ & $\begin{array}{l}48.6 \text { at } \\
\text { baseline }\end{array}$ & $\begin{array}{l}\text { LupusQoL } \\
\text { Physical health, pain, plan- } \\
\text { ning, burden to others, } \\
\text { emotional health, fatigue }\end{array}$ \\
\hline $\begin{array}{l}\text { Poomsalood } \\
\text { et al }{ }^{\star}\end{array}$ & Thailand & 237 & $\begin{array}{c}\text { SLEDAI } \leq 2 \\
\text { Serologic= } \\
\text { allowed } \\
\text { PGA=NR } \\
\text { PDN } \leq 7.5 \\
\text { IS=Yes } \\
\text { AM=Yes } \\
\text { New manifesta- } \\
\text { tions: NR } \\
\text { Duration=NR }\end{array}$ & 61.6 & $\begin{array}{c}\text { SLEQoL } \\
\text { Univariable: } \\
\text { physical, activities, symp- } \\
\text { tom, treatment, mood, } \\
\text { self-image and total. } \\
\text { Multivariable: Better global } \\
\text { QoL }\end{array}$ \\
\hline
\end{tabular}

PCS: Physical component summary. MCS: Mental component summary. ${ }^{*}$ Cross-sectional \#Longitudinal

Table 1. Association between remission and HRQoL

\begin{tabular}{|c|c|c|c|c|c|}
\hline Authors & $\begin{array}{l}\text { Country/ } \\
\text { Region }\end{array}$ & Patients & Remission & $\begin{array}{l}\text { Remission } \\
(\%)\end{array}$ & $\begin{array}{l}\text { Domains positively associated } \\
\text { or predicted by remission }\end{array}$ \\
\hline Mok et al ${ }^{*}$ & China & 769 & $\begin{array}{c}\text { SLEDAI }=0 \\
\text { Serologic= } \\
\text { Allowed } \\
P G A<0.5 \\
P D N \leq 5 \\
I S=Y e s \\
A M=Y e s \\
\text { Duration } \geq 5 \text { years }\end{array}$ & 25.1 & $\begin{array}{c}\text { Remission }>5 \text { years vs not on } \\
\text { remission } \\
\text { SF-36: } \\
\text { Role physical, bodily pain, } \\
\text { general health, vitality, } \\
\text { social functioning, role } \\
\text { emotional, PCS and MCS } \\
\text { LupusPRO: } \\
\text { Symptoms, medications, pro- } \\
\text { creation, physical health, } \\
\text { pain, emotional, image, } \\
\text { HRQoL total }\end{array}$ \\
\hline $\begin{array}{l}\text { Tsang-A- } \\
\text { Sjoe et } \\
\text { al\# }\end{array}$ & Netherlands & 154 & $\begin{array}{c}\text { SLEDAI=0 } \\
\text { Serologic= } \\
\text { allowed } \\
\text { PGA } \leq 2 / 10 \\
\mathrm{PDN} \leq 5 \\
\mathrm{IS}=\text { Yes } \\
\mathrm{AM}=\text { Yes } \\
\text { Duration }=\mathrm{NR}\end{array}$ & $\begin{array}{l}39.0 \text { at } \\
\text { baseline }\end{array}$ & $\begin{array}{l}\text { Remission on- or off-therapy } \\
\text { predicted a better SF-36 } \\
\text { PCS but not MCS }\end{array}$ \\
\hline $\begin{array}{c}\text { Margiotta } \\
\text { et } \mathrm{al}^{\star}\end{array}$ & Italy & 136 & $\begin{array}{c}\text { SLEDAI=0 } \\
\text { Serologic= } \\
\text { allowed } \\
\text { PGA }=\text { NR } \\
P D N \leq 5 \\
I S=Y e s \\
A M=Y e s \\
\text { Duration }>5 \text { years }\end{array}$ & 39.0 & $\begin{array}{c}\text { Remission }>5 \text { years vs unre- } \\
\text { mitted or remission }<5 \text { years } \\
\text { SF-36: } \\
\text { Physical health, role physical, } \\
\text { bodily pain, general health, } \\
\text { social functioning }\end{array}$ \\
\hline
\end{tabular}

PCS: Physical component summary. MCS: Mental component summary. ${ }^{*}$ Cross-sectional \#Longitudinal

Alarcon: None declared, Bernardo Pons-Estel Grant/research support from: GSK, Janssen, Consultant of: GSK, Janssen, Speakers bureau: GSK, Janssen

DOI: 10.1136/annrheumdis-2020-eular.3995 